\title{
Fragilidad cognitiva, un desafío en evolución
} Cognitive Frailty, an Evolving Challenge

\author{
Estephania Chacón-Valenzuela \\ Pontificia Universidad Javeriana, Colombia \\ Elly Morros-González \\ Pontificia Universidad Javeriana, Colombia \\ María Paula Vargas Beltrán \\ Pontificia Universidad Javeriana, Colombia \\ Luis Carlos Venegas-Sanabria \\ Pontificia Universidad Javeriana, Colombia \\ Ronald Camilo Gómez Arteaga \\ Univ. Ciencias Aplicadas y Ambientales, \\ Colombia \\ Diego Chavarro Carvajal \\ Pontificia Universidad Javeriana, Colombia \\ Hospital Universitario San Ignacio, Colombia \\ Carlos Cano-Gutiérrez \\ Pontificia Universidad Javeriana, Colombia \\ Hospital Universitario San Ignacio, Colombia
}

Recepción: 05/12/2018 | Aceptación: 26/02/2019
Cómo citar: Chacón-Valenzuela E, Morros-González E, Vargas-Beltrán M, Venegas-Sanabria L, GómezArteaga RC, Chavarro Carvajal D, Cano Gutiérrez CA. Fragilidad cognitiva, un desafío en evolución. Univ. Med. 2019;60(3). https://doi.org/10.11144/Jave riana.umed60-3.fcde

\section{RESUMEN}

Dentro de los principales desafíos relacionados con el envejecimiento se encuentran la fragilidad y el deterioro cognitivo, condiciones que se han relacionado con aumento en la declinación funcional, la discapacidad y el deterioro en la calidad de vida. Durante los últimos años se ha demostrado la relación bidireccional entre estas dos condiciones clínicas y dentro del Grupo de Consenso Internacional en Toulouse, Francia, surgió el término fragilidad cognitiva, en el 2013. Ese estado reversible se ha considerado un nuevo síndrome geriátrico, caracterizado por la coexistencia entre fragilidad física y deterioro cognitivo; sin embargo, aún sin criterios diagnósticos definidos. El objetivo es realizar una aproximación de la definición, fisiopatología, herramientas diagnósticas y su respectiva asociación con desenlaces adversos, para realizar un diagnóstico oportuno y aplicar intervenciones multidominio centradas en la actividad física, el enfoque nutricional y el entrenamiento cognitivo, que puedan modificar el curso de este estado dinámico y reversible, previniendo el deterioro funcional y mejorando la calidad de vida de las personas mayores.

Palabras clave

fragilidad; cognición; adulto mayor; anciano; movilidad; memoria.

\begin{abstract}
The main challenges facing the increase of elderly population are the frailty and cognitive impairment, which are related to increasing disability, worse quality of life and cognitive decline. During the last years, it has been proved the two-way relationship between frailty and cognitive impairment, hence the concept cognitive frailty emerged by an international consensus group on 2013 in Toulouse (France). This reversible stage has been considered a new geriatric syndrome characterized by the coexistence between physical frailty
\end{abstract}


and cognitive impairment, however, there is no consensus regarding diagnostic criteria. The objective of this study is to make an approach to the definition pathophysiology, and diagnostic instruments, and their respective association with adverse outcomes, to make an opportune diagnosis and apply multi-domain interventions focused on physical activity, nutritional focus and cognitive training, which can modify the course of this dynamic and reversible state by preventing functional impairment and improving the quality of life for the elderly.

Keywords

frailty; memory; motility; elderly; cognition.

\section{Introducción}

Debido al fenómeno de transición demográfica, se han producido grandes cambios en la pirámide poblacional, con un aumento en la expectativa de vida y una alta carga de enfermedades crónicas no transmisibles y estados de discapacidad $(1,2)$ que han convertido el envejecimiento en un desafío para la sociedad moderna, ya que enfermedades como insuficiencia cardiaca, insuficiencia renal, inflamación crónica, alteraciones nutricionales, enfermedades vasculares (3), entre otras, disminuyen las reservas fisiológicas, lo que lleva a un desequilibrio homeostático o fragilidad (4). Esto ha hecho que se desarrollen herramientas para la detección temprana de estados reversibles, tanto físicos como cognitivos — por ejemplo, la detección de dinapenia y la disminución de fuerza muscular, que han demostrado influir en la presencia de deterioro cognitivo, así como la disminución de la marcha, y lentitud en el lenguaje - variables que pueden ser reversibles con entrenamiento multidominio (3).

También se han originado nuevos conceptos, como fragilidad, definida como la disminución de la reserva fisiológica que lleva al desequilibrio homeostático y genera un estado de vulnerabilidad ante factores estresores (5) y deterioro cognitivo leve, definido como la alteración de uno o más dominios cognitivos que no interfieran en la funcionalidad (6). Por ello, últimamente, se ha planteado la relación bidireccional entre la fragilidad y la disfunción cognitiva, y ha surgido el término fragilidad cognitiva (figura 1). Se han propuesto diferentes criterios según la patogénesis, incluyendo desde medidas físicas, clínicas y de ejecución, hasta acúmulo de déficits en los que pueden estar presentes los dominios cognitivos $(7,8)$.

\section{Figura 1}

Representación de la fragilidad cognitiva

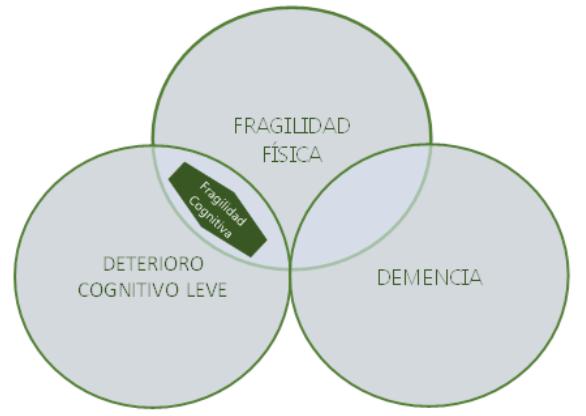

Existe un gran debate sobre la definición precisa y no existe un consenso específico respecto a los criterios diagnósticos; sin embargo, en 2013, el Grupo de Consenso Internacional en Toulouse, Francia, estableció la primera definición operacional, al considerarla un síndrome geriátrico reversible y heterogéneo, caracterizado por la coexistencia de fragilidad física y deterioro cognitivo (9). Posteriormente, surgieron hipótesis que relacionan estas dos condiciones como un círculo vicioso, evidenciándose mayor riesgo de alteraciones en dominios cognitivos en ancianos con limitaciones físicas (10) y, así mismo, mayor riesgo de deterioro funcional en aquellos que presentan deterioro cognitivo (11).

Adicionalmente, hay que considerar el efecto económico en los sistemas sanitarios, que no se incrementan únicamente por el aumento de la población adulta mayor, sino también porque personas que presentan deterioro en su funcionalidad, relacionados con estados de fragilidad y discapacidad grave, usan los recursos en salud y hay un aumento en estas condiciones en los años previos a la muerte (12).

El objetivo del presente trabajo es describir el concepto de fragilidad cognitiva y su implicación en la práctica clínica diaria, haciendo hincapié en su definición y criterios diagnósticos. 


\section{Metodología}

El presente trabajo se basa en la revisión de la literatura disponible sobre el concepto de fragilidad cognitiva, que se presenta a modo de revisión narrativa.

\section{Definición}

La fragilidad se ha definido de diferentes formas, aunque en general es considerada un estado de vulnerabilidad reversible, multidimensional, secundario a la disminución de la reserva fisiológica, que lleva a una pobre respuesta ante factores estresores agudos (5). El deterioro cognitivo se define como la alteración en uno o más de los dominios cognitivos que, dependiendo de la severidad, puede o no interferir en el funcionamiento de la vida diaria (6). Dada la asociación entre fragilidad física y deterioro cognitivo en personas mayores, se han realizado diversos estudios que demuestran su asociación.

En 2001, Paganini-Hill et al. (13) fueron los primeros en hablar de este término, proponiendo la utilidad de la prueba del reloj para predecir fragilidad cognitiva. Posteriormente, Panza et al. (14), en 2006, utilizaron este término como factor de riesgo en una fase prodrómica de demencia vascular; sin embargo, hasta 2013, un grupo compuesto por el International Academy of Nutrition and Aging (IANA) y el International Association of Gerontology and Geriatrics (IAGG) definieron la fragilidad cognitiva de forma operacional como la coexistencia de fragilidad física, utilizando el modelo de fenotipo propuesto por Linda Fried, y deterioro cognitivo leve, definido como Clinical Dementia Rating Score (CDR) de 0,5, en ausencia de diagnóstico de demencia (9). Sin embargo, esta definición presenta una limitación, ya que puede llegar a excluir a la población que presenta etapas tempranas de fragilidad cognitiva, por lo que los criterios diagnósticos aún no están específicamente establecidos.

\section{Epidemiología}

La prevalencia de fragilidad oscila entre el 4,0\% y el $59,1 \%$ en personas no institucionalizadas, dependiendo de la definición y las herramientas con la que se evalúe (15). La prevalencia de deterioro cognitivo leve es del 10\% al 20\%, dependiendo del entorno en que se realiza el diagnóstico (16).

En los últimos años, estudios transversales han evaluado la prevalencia de fragilidad cognitiva en la comunidad y han encontrado una prevalencia entre un $1 \%$ y un $5 \%$ (3). Shimada et al. (17), en un estudio en 5104 personas mayores de 65 años, con edad media de 71 años, evidenciaron una prevalencia de fragilidad cognitiva del 2,7\% (17); posteriormente, se realizaron otros estudios, entre estos, en 2017, el de Roppolo et al. (18), que evaluó a 594 sujetos mayores, con una edad media de 73,6 años. Este documentó una prevalencia de fragilidad cognitiva del $4,4 \%$.

\section{Impacto}

La importancia de la fragilidad cognitiva está dada por su asociación con una declinación funcional, discapacidad, empeoramiento en la calidad de vida, mayor número de ingresos hospitalarios y aumento en mortalidad $(10,17)$. Feng et al. (7), en un estudio longitudinal de 3 años, de 2375 personas mayores de 55 años en Singapur, con edad media de 66 años, encontraron que la fragilidad cognitiva se asocia a mayor deterioro en la calidad de vida (OR: 26,9; IC95\%: 3,05-238,4; p $\leq$ 0,003), y 5 veces mayor riesgo de mortalidad (HR: 5,12; IC95\%: 3,00-8,74; $\mathrm{p}<0,001)$. En otro estudio longitudinal, Solfrizzi et al. (11) demostraron que la fragilidad cognitiva es un predictor a corto plazo (3,5 años) y largo plazo (7,0 años) de mortalidad. Del mismo modo, Yunhwan Lee y colaboradores compararon personas sin fragilidad ni deterioro cognitivo con aquellos que sí lo presentaban y encontraron una tasa de supervivencia a 3 años menor en el último grupo (HR: 1,92; IC95\%: 1,26-2,93; $\mathrm{p}=0,003$ ), por lo que se consideró que fragilidad física y 
deterioro cognitivo son predictores significativos de mortalidad a 3 años en adultos mayores no institucionalizados (19).

\section{Fisiopatología}

Todavía se discute respecto a si la fragilidad física precede o es consecuencia del deterioro cognitivo. Se han tratado de dilucidar vías comunes, incluyendo el proceso inflamatorio sistémico, alteraciones hormonales relacionadas con cambios fisiológicos del envejecimiento, factores de riesgo cardiovascular, cambios nutricionales y alteraciones en el cerebro.

\section{Estrés oxidativo e inflamación}

El cerebro parece ser deficiente en cuanto a mecanismos protectores ante radicales libres; adicionalmente, usa altas cantidades de oxígeno y tiene facilidad para que los ácidos grasos realicen su proceso de peroxidación, por lo que el estrés oxidativo puede aumentar el riesgo de deterioro cognitivo (20). Este no solo se relaciona con neurodegeneración, sino que también interviene en la señalización de receptores de insulina, regulando la baja actividad proteolítica autofágica de las células, que normalmente contribuye a la homeostasis celular, y de la misma manera con la vía de la sirtuina, relacionada con la regulación de procesos inflamatorios inducidos por el factor de necrosis tumoral alfa (TNF-alfa), regulación de la vía de señalización de la insulina y control de niveles de especies reactivas de oxígeno (21). A nivel sistémico, el estrés oxidativo ocasiona daño del músculo esquelético y se relaciona con el desarrollo de sarcopenia (20), que podría estar relacionado con el aumento del riesgo de fragilidad física.

Se ha descrito que el estado proinflamatorio en adultos mayores aumenta 4,06 veces la probabilidad de presentar fragilidad en el seguimiento a un año (22). En el proceso inflamatorio compartido por la fragilidad y el deterioro cognitivo se encuentran cantidades elevadas de interleucina 8 (IL-8), interleucina 6
(IL-6), proteína C reactiva (PCR) y TNF-alfa. Estas concentraciones elevadas de citocinas se asocian con un bajo desempeño en la memoria y en otros dominios cognitivos, así como a una lentificación de la función motora. Se habla de IL-6 y el TNF-alfa como biomarcadores de fragilidad, y los niveles altos de PCR e IL-6 como biomarcadores de deterioro cognitivo $(23,24,25,26,27,28,29)$. Adicionalmente, se ha descrito que el estrés oxidativo aumenta el riesgo de deterioro cognitivo y fragilidad, interrumpiendo procesos de señalización del receptor de insulina y actividades proteolíticas autofágicas de la célula que en condiciones normales contribuyen con la homeostasis celular $(21,28)$.

Acumulación de proteína b-amiloide en sistema nervioso

En cuanto a los hallazgos en el sistema nervioso central, la evaluación de la función del lóbulo frontal resulta importante para el diagnóstico de fragilidad cognitiva, puesto que las personas con esta condición tienden a presentar mayor deterioro en la función ejecutiva (velocidad de procesamiento, atención selectiva y flexibilidad mental), en comparación con aquellos con deterioro cognitivo sin fragilidad física (30). Se han propuesto diferentes teorías como la acumulación de proteína $\beta$-amiloide, que en localizaciones específicas se ha asociado con deterioro en la velocidad de la marcha por efecto neurotóxico $(27,31,32)$. Asimismo, la proteína amiloide promueve el depósito de proteína tau y esta se ha relacionado con disfunción sináptica y, por consiguiente, pérdida neuronal $(27,33)$.

Gracias a la tomografía por emisión de positrones se ha descrito una asociación entre la velocidad de la marcha y la acumulación de proteína $\beta$-amiloide, en la región anterior y posterior del putamen, la corteza occipital, el precuneus y el cíngulo anterior, de forma independiente a la edad, genotipo de APOE y estadio de enfermedad.

Adicionalmente, se ha visto que la asociación entre proteína $\beta$-amiloide y la velocidad de la 
marcha puede estar relacionada con actividad física, tabaquismo, enfermedades metabólicas y cardiovasculares; sin embargo, no se ha establecido claramente su causalidad (31).

Se ha propuesto que personas con actividad física regular que presentan aumento en la velocidad de la marcha tienen depósitos de proteína $\beta$-amiloide significantemente menores, en comparación con aquellas personas sedentarias, con velocidad de la marcha disminuida. Asimismo, el depósito de esta proteína en fibras musculares se asocia a miositis por cuerpos de inclusión, lo que disminuye la fuerza muscular, lo que aumenta el riesgo de sarcopenia y fragilidad (32).

Enfermedades con riesgo cardiovascular y disfunción endotelial

Se ha encontrado asociación entre enfermedades cardiovasculares, como falla cardiaca, enfermedad coronaria, enfermedad arterial periférica, fibrilación auricular, diabetes mellitus e hipertensión arterial, con la presencia de fragilidad $(34,35)$, dado por la alteración del sistema endotelial y aumento de especies reactivas de oxígeno. Esta asociación se ha relacionado con la confluencia de los mecanismos que contribuyen a un estado proinflamatorio, eventos embólicos y enfermedad ateroesclerótica, los cuales disminuyen el flujo sanguíneo de forma multisistémica, aumentando el riesgo de deterioro cognitivo (1).

\section{Desbalance hormonal}

Por los cambios fisiológicos del envejecimiento, como la resistencia a la insulina, el aumento de cortisol y los niveles bajos de testosterona y hormona de crecimiento, se ha evidenciado mayor riesgo de deterioro cognitivo y fragilidad física $(1,27,36,37,38)$. Dentro del desbalance hormonal más estudiado, la disminución de niveles de testosterona y andrógenos se pueden relacionar con el desarrollo de fragilidad y deterioro cognitivo, puesto que en condiciones normales promueven la plasticidad neuronal en el hipocampo y regulan la acumulación de proteína $\beta$-amiloide (39). Igualmente, niveles bajos de esta hormona se relacionan con deterioro de masa muscular, lo que aumenta también el riesgo de desarrollar sarcopenia y fragilidad $(27,40)$.

\section{Criterios diagnósticos}

\section{Fragilidad}

La fragilidad es un estado previo a la discapacidad y su importancia radica en la posibilidad de una intervención temprana. Diversos estudios han aplicado diferentes criterios para definir fragilidad. Dentro de los modelos descritos, la definición más utilizada y validada para la investigación es el fenotipo propuesto por Linda Fried (7). Este modelo describe la fragilidad desde el dominio físico y se fundamenta en el cumplimiento de tres de los siguientes criterios: pérdida de peso involuntaria, fatiga, disminución en la velocidad de la marcha, disminución en fuerza de prensión y baja actividad física (7). Adicionalmente, Fried describió un estado intermedio, definido como el cumplimiento de uno o más criterios. Este estado intermedio ahora se conoce como prefragilidad.

Un segundo modelo aborda la fragilidad como un acúmulo de déficits que va más allá del dominio físico, incluyendo el dominio clínico y psicosocial, conocido como el índice de fragilidad $(41,42)$. Para el cálculo de este índice se incluye, además, el estado cognitivo, la multimorbilidad e, incluso, la presencia de discapacidad.

Como combinación de los anteriores modelos de fragilidad, surge a partir del IANA, la escala FRAIL, que incluye los siguientes criterios: fatiga, resistencia, deambulación, comorbilidades y pérdida de peso; las puntuaciones de la escala varían de 0 a 5 puntos, siendo mayor de 3 un estado de salud frágil $(43,44,45)$. Otro instrumento es el cuestionario autoinforme de 15 preguntas que evalúan los tres dominios (físicos, psicológicos y sociales), citado como indicador de fragilidad de Tilburg, donde los rangos de 
puntuación más altos indican un mayor riesgo de fragilidad (46).

Adicionalmente, se ha propuesto la aplicación de una prueba de ejecución física para evaluar la fragilidad, prueba que ya se encuentra validada en Colombia (47). Esta es el Short Physical Performance Battery (SPPB) o Test de Guralnik. Se trata de un constructo de tres subpruebas, que incluyen la evaluación del equilibrio en bipedestación, posteriormente la velocidad de la marcha en cuatro metros a paso usual y, finalmente, levantarse de una silla cinco veces de forma consecutiva y sin utilizar los miembros superiores como apoyo. Cada subprueba puntúa desde 0 , como peor desempeño, hasta 4, como el mejor desempeño, y el puntaje final de la prueba resulta de la sumatoria de los resultados de las 3 subpruebas, variando de 0 a 12, donde un resultado menor o igual a 9 se relaciona con un alto riesgo de desarrollar discapacidad, convirtiéndose en punto de corte a nivel mundial para considerar frágil a un anciano $(48,49)$.

\section{Deterioro cognitivo}

El deterioro cognitivo se define como la alteración en uno o más de los dominios cognitivos (atención compleja, memoria de aprendizaje, lenguaje, función ejecutiva, funciones perceptuales y psicomotoras $y$ cognición social) respecto al desempeño esperado para la edad y el nivel educativo, reconocida por el paciente, la familia o por observación propia del médico (6).

De acuerdo con las presentaciones, se definieron tres etapas (9):

1. Etapa preclínica y asintomática: donde el uso de biomarcadores cumple un papel fundamental. Los más estudiados son la proteína $\beta$-amiloidea y la proteína tau, que reflejan de forma directa la patología por presencia de depósitos de estas proteínas (50). Hay biomarcadores de daño neuronal $o$ procesos neurodegenerativos, como los niveles de proteína tau y tau fosforilada en líquido cefalorraquídeo (27). También hay biomarcadores relacionados con la muerte neuronal, estrés oxidativo e inflamación, como IL- 6 , TGF- $\beta$, TNF- $\alpha$, e IL1- $\beta$ (50).

2. Etapa predemencial: caracterizada por el deterioro de la memoria u otros dominios cognitivos, preservando la independencia en las habilidades funcionales y sin afectación de las actividades sociales u ocupacionales. Esta etapa se conoce como deterioro cognitivo leve.

3. Etapa de demencia: caracterizada por el deterioro en el rendimiento en los dominios cognitivos, que interfiere significativamente con la capacidad de vivir de forma independiente.

Sin embargo, en la actualidad, el diagnóstico del deterioro cognitivo se realiza en etapas avanzadas de demencia por la falta de conocimiento de las diferentes herramientas de evaluación que permiten la identificación temprana (9). Por esta razón, las investigaciones se encuentran enfocadas en la identificación de la fase predemencial, surgiendo nuevas herramientas como la realización de escalas autoinformadas o informadas de queja subjetiva de memoria para la detección precoz de esta condición (51).

\section{Interacción entre la fragilidad y deterioro cognitivo}

En 2013, un grupo compuesto por expertos del IANA y la IAGG definieron la fragilidad cognitiva como la coexistencia de fragilidad física, utilizando el modelo de fenotipo propuesto por Linda Fried, previamente descrito, y el deterioro cognitivo, medido con CDR, desarrollado por Hughes et al. (52), en ausencia de diagnóstico de demencia (9), como se puede ver en la tabla 1. 


\section{Tabla 1}

Comparación de criterios de fragilidad cognitiva según el consenso en Toulouse, 2013, y criterios propuestos por Won et al.

\begin{tabular}{|l|l|}
\hline \multicolumn{1}{|c|}{$\begin{array}{c}\text { Criterios de fragilidad } \\
\text { cognitiva (Consenso } \\
\text { Toulouse de 2013) }\end{array}$} & \multicolumn{1}{|c|}{$\begin{array}{c}\text { Criterios fragilidad } \\
\text { cognitiva (Won et al., } \\
\text { 2018) }\end{array}$} \\
\hline $\begin{array}{l}\text { 1. Presencia de fragilidad } \\
\text { física }\end{array}$ & $\begin{array}{l}\text { 1. Presencia de fragilidad } \\
\text { física }\end{array}$ \\
\hline $\begin{array}{l}\text { 2. Deterioro cognitivo por } \\
\text { escala CDR }=0,5\end{array}$ & $\begin{array}{l}\text { 2. Deterioro cognitivo: 1,5 } \\
\text { desviaciones estándar del } \\
\text { promedio en cualquier } \\
\text { prueba de función } \\
\text { cognitiva* }\end{array}$ \\
\hline $\begin{array}{l}\text { 3. Exclusión de concurrencia } \\
\text { de demencia tipo Alzheimer } \\
\text { u otro tipo de demencia }\end{array}$ & $\begin{array}{l}\text { 3. No dependencia en } \\
\text { actividades Instrumentales } \\
\text { de la vida diaria** }\end{array}$ \\
\hline
\end{tabular}

\Criterios Fragilidad L. Fried.

*Ajustado por sexo, edad y nivel educativo.

**Incluyendo: manejo de dinero, uso de teléfono, responsabilidad para toma de medicamentos y mantener su agenda de actividades.

El CDR permite evaluar la gravedad del deterioro cognitivo, considerando como criterio para la fragilidad cognitiva un resultado igual a 0,5 (tabla 2). Sin embargo, resulta complejo el uso de esta clasificación en estudios epidemiológicos, ya que la mayoría se basan en información de entrevistas semiestructuradas que evalúan los dominios cognitivos, para el 2018, Chan Won Won et al. (53) desarrollaron una nueva definición de fragilidad cognitiva para estudios epidemiológicos (tabla 1), incluyendo fragilidad física y deterioro cognitivo, considerado como 1,5 desviaciones estándar por debajo del promedio en la prueba de función cognitiva, ajustado por edad, sexo y nivel educativo, con cualquiera de los siguientes test: Montreal Cognitive Assesment Test, Alzheimer's Disease Assessment Scale-Cognitive Subscale (ADAS-Cog), Verbal Learning Test, Digit Span, Boston Naming Test, Trail Making Test y Frontal Assessment Battery. Adicionalmente, la persona no debe tener dependencia en actividades instrumentales de la vida diaria, especialmente en manejo de dinero, uso de teléfono, responsabilidad para toma de medicamentos y mantener su agenda de actividades, ya que son tareas que requieren mayores competencias cognitivas (53).
Tabla 2

Valoración clínica de la demencia según la escala CDR con 0,5 puntos

\begin{tabular}{ll|}
$\begin{array}{l}\text { Valoración Clínica de } \\
\text { Demencia (CDR) }\end{array}$ & \multicolumn{1}{c|}{$\mathbf{0 , 5}$ puntos } \\
Memoria & $\begin{array}{l}\text { Olvidos leves constantes, recolección } \\
\text { parcial de eventos olvidos "benignos". } \\
\text { Completamente orientado, pero con leve } \\
\text { dificultad para las relaciones temporales. } \\
\text { Orientación }\end{array}$ \\
$\begin{array}{l}\text { Razonamiento y dicultad para resolver problemas, } \\
\text { solución de problemas } \\
\text { Actividades fuera de } \\
\text { casa }\end{array}$ & $\begin{array}{l}\text { Leve dificultad en estas actividades. } \\
\text { Actividades domésticas diferencias. } \\
\text { y aficiones }\end{array}$ \\
$\begin{array}{l}\text { Vida en casa, aficiones e intereses } \\
\text { intelectuales algo limitados. } \\
\text { Cuidado personal }\end{array}$ & $\begin{array}{l}\text { Completamente capaz de cuidarse por sí } \\
\text { misma. }\end{array}$
\end{tabular}

\section{Intervención}

La fragilidad, entendida como un síndrome, exige un enfoque multidimensional para su manejo (54). La intervención multidimensional se define como aquella en la que se trabaja sobre, por lo menos, dos dominios de la valoración geriátrica integral (cognitivo, nutricional, funcional $\mathrm{O}$ social) (55). Actualmente, las más efectivas involucran el ejercicio y las intervenciones nutricionales; sin embargo, no se puede afirmar que estas sean las únicas efectivas, puesto que no se han realizado estudios que involucren otras intervenciones, como sociales, cognitivas o psicológicas. Además, la falta de unificación dificulta la comparación entre los estudios realizados (55).

\section{Ejercicio}

Se recomienda como intervención realizar ejercicio tres veces por semana de intensidad moderada progresiva, de 30 a 45 minutos por sesión, con una duración mínima de 5 meses. Se debe tener en cuenta que la intervención debe ser multicomponente, para que se obtenga el mayor efecto en la fragilidad cognitiva. La intervención multicomponente incluye ejercicio aeróbico, entrenamiento de fuerza, equilibrio y flexibilidad. Esta intervención ha evidenciado ser efectiva en la mejoría de las funciones físicas, 
depresión, disminución de caídas y cognición (54).

\section{Nutrición}

El enfoque del manejo nutricional debe ser individualizado según los hábitos alimentarios, estado nutricional y capacidades económicas, realizando ajustes durante la intervención para obtener los resultados deseados (54). Se ha demostrado que el $50 \%$ de las personas con fragilidad cursan con desnutrición, y el $90 \%$, con riesgo de malnutrición (55).

Como intervenciones principales se ha evidenciado que la ingesta de proteínas entre 1,2 y $1,5 \mathrm{~g} / \mathrm{kg}$ cada día se relaciona con menor riesgo para desarrollar fragilidad (54). Además, se ha demostrado que la adherencia a una dieta mediterránea, rica en alimentos antioxidantes, disminuye el riesgo de fragilidad y de deterioro de la función cognitiva $(27,28,56)$.

\section{Otras intervenciones}

Como intervenciones primarias, el abandono del hábito de fumar, la promoción de la recuperación emocional, la participación en un estilo de vida activo y socialmente integrado, una cantidad ideal de sueño diario, el mantenimiento de peso corporal adecuado y el control metabólico influyen significativamente en la fragilidad cognitiva (27).

Como intervenciones secundarias, el abordaje de síndromes geriátricos, revisión de polifarmacia, prevención de caídas e intervenciones sociales tienen un impacto positivo en la fragilidad cognitiva; sin embargo, hasta el momento no se han diseñado estudios clínicos para evaluar su efecto real $(54,55)$.

Finalmente, la intervención multidominio, enfocada en dieta, ejercicio, entrenamiento cognitivo y reducción de factores de riesgo cardiovascular, previenen el deterioro cognitivo en personas mayores (57).

\section{Conclusión}

La fragilidad cognitiva, como estado reversible previo a la discapacidad y a la demencia, se convierte en un desafío para la práctica médica, ya que su adecuada evaluación permite la intervención en etapas previas a la declinación funcional o cognitiva. Sin embargo, sus criterios diagnósticos aún son controvertidos. En cuanto a las intervenciones planteadas, el manejo nutricional y la prescripción de ejercicio multimodal son las más efectivas sobre la fragilidad cognitiva. En los últimos años se ha visto un mayor interés en el mundo por investigar y crear evidencia científica en el tema; sin embargo, se requieren estudios en el país que identifiquen y caractericen variables asociadas con esta condición.

\section{Referencias}

1. Azzopardi RV, Beyer I, Vermeiren S, Petrovic M, Noortgate N Van Den. Increasing use of cognitive measures in the operational definition of frailty: A systematic review. Ageing Res Rev. 2018;43:10-6.

2. Dulcey E, Arrubla D, Sanabria P. Envejecimiento y vejez en Colombia. Vol. 6. Bogotá: Profamilia Colombia; 2013.

3. Hidenori A, Stake S, Kozaki K. Cognitive frailty in geriatrics aging frailty mild cognitive impairment dementia disability exercise. Clin Geriatr Med. 2018;34(4):667-75.

4. Clegg A, Young J, Iliffe S, Rikkert MO, Rockwood K. Frailty in elderly people. Lancet. 2013;381(9868):752-62.

5. Morley J, Vellas B, Kan G van, Anker S. Frailty consensus: a call to action. J Am. 2013;14(6):392-7.

6. American Psychiatric Association. DSM-5: Manual diagnóstico y estadístico de los trastornos mentales. 
5a ed. Madrid: Editorial Médica Panamericana; 2014. p. 319-37.

7. Fried LP, Tangen CM, Walston J, et al. Frailty in older adults: evidence for a phenotype. J Gerontol Ser A Biol Sci Med Sci. 2001;56(3):M146-57.

8. Rockwood K, Bergman H. Frailty: a report from the 3rd Joint Workshop of IAGG/WHO/SFGG, Athens, January 2012. Can Geriatr J. 2012;15(2):31-6.

9. Kelaiditi E, Cesari M, Canevelli M, et al. Cognitive frailty: rational and definition from an (I.A.N.A/I.A.G.G) International consensus group. J Nutr Heal Aging. 2013;17(9):726-34.

10. Feng L, Zin Nyunt MS, Gao Q, et al. Cognitive frailty and adverse health outcomes: findings from the Singapore Longitudinal Ageing Studies (SLAS). J Am Med Dir Assoc. 2017;18(3):252-8. 11. Solfrizzi V, Scafato E, Lozupone $\mathrm{M}$, et al. Additive role of a potentially reversible cognitive frailty model and inflammatory state on the risk of disability: the Italian Longitudinal Study on Aging. Am J Geriatr Psychiatry. 2017;25(11):1236-48.

12. Morley JE. Cognitive frailty: a new geriatric syndrome? Eur Geriatr Med. 2015;6(5):408-11.

13. Paganini-Hill A, Clark LJ, Henderson V, Birge S. Clock drawing: analysis in a retirement community. J Am Geriatr Soc. 2001;49(7):941-7.

14. Panza F, Solfrizzi V, Barulli MR, et al. Cognitive frailty - epidemiological and neurobiological evidence of an age-related clinical condition: a systematic review. Rejuvenation Res. 2015;18(5):389-412.

15. Collard RM, Boter H, Schoevers RA, Oude Voshaar RC. Prevalence of frailty in community-dwelling older persons: a systematic review. J Am Geriatr Soc. 2012;60(8):1487-92.
16. Langa KM, Levine DA. The diagnosis and management of mild cognitive impairment: A clinical review. JAMA. 2014;312(23):2551-61. 17. Shimada H, Makizako $H$, Lee $S$, et al. Impact of cognitive frailty on daily activities in older persons. J Nutr Heal Aging. 2016;20(7):729-35.

18. Roppolo M, Mulasso A, Rabaglietti E. Cognitive frailty in italian community-dwelling older adults: Prevalence rate and its association with disability. J Nutr Health Aging. 2017;21(6):631-6.

19. Lee Y, Kim J, Chon D, Lee KE, Kim JH, Myeong S, et al. The effects of frailty and cognitive impairment on 3-year mortality in older adults. Maturitas. 2018;107:50-5.

20. Mulero J, Zafrilla P, MartinezCacha A. Oxidative stress, frailty and cognitive decline. J Nutr Health Aging. 2011;15(9):756-60.

21. Liang F, Kume S, Koya D. SIRT1 and insulin resistance. Nat Rev Endocrinol. 2009;5(7):367-73.

22. Hsieh $\mathrm{T}$, Chang $\mathrm{H}$, Wu I, et al. Independent association between subjective cognitive decline and frailty in the elderly. PLoS One. 2018;13(8):1-12.

23. Yaffe $\mathrm{K}$, Lindquist $\mathrm{K}$, Penninx $\mathrm{BW}$, et al. Inflammatory markers and cognition in well-functioning AfricanAmerican and white elders. Neurology. 2003;61(1):76-80.

24. Hubbard RE, Woodhouse KW. Frailty, inflammation and the elderly. Biogerontology. 2010;11:635-41.

25. Michaud M, Balardy L, Moulis G, et al. Proinflammatory cytokines, aging, and age-related diseases. J Am Med Dir Assoc. 2013;14(12):877-82.

26. Collerton J, Martin-ruiz C, Davies $\mathrm{K}$, et al. Frailty and the role of inflammation, immunosenescence and 
cellular ageing in the very old: Crosssectional findings from the Newcastle 85 + Study. Mech Ageing Dev. 2012;133(6):456-66.

27. Fougère B, Delrieu J, del Campo $\mathrm{N}$, et al. Cognitive frailty: mechanisms, tools to measure, prevention and controversy. Clin Geriatr Med. 2017;33(3):339-55.

28. Mulero J, Zafrilla P, MartínezCacha A. Oxidative stress, frailty and cognitive decline. J Nutr Health Aging. 2011;15(9):756-60.

29. Ershler WB, Keller ET. Ageassociated increased interleukin- 6 gene expression, late-life disease, and frailty. Annu Rev Med. 2000;51:245-70.

30. Delrieu J, Andrieu S, Pahor $\mathrm{M}$, et al. Neuropsychological profile of "cognitive frailty" subjects in MAPT Study. J Prev Alzheimers Dis. 2016;3(3):151-9.

31. del Campo N, Payoux P, Djilali A, et al. Relationship of regional brain b-amyloid to gait speed. Am Acad Neurol. 2015;86:1-8.

32. Yoon DH, Lee J-Y, Shin SA, Kim YK, Song W. Physical frailty and amyloid- $\beta$ deposits in the brains of older adults with cognitive frailty. J Clin Med. 2018;7 (169):1-12.

33. Gómez-Isla T, Hollister R, West $\mathrm{H}$, et al. Neurond loss correlates with but exceeds neurofibrillary tangles in Alzheimer's disease. Am Neurol Assoc. 1997;41:17-24.

34. Chainani V, Riehl R, Chainani G, et al. Frailty and cardiovascular disease.

En: Frailty and sarcopenia-onset, development and clinical challenges. Intech Open [internet]; 2017. p. 196-209. https://doi.org/10.5772/6515 3.

35. Sinclair AJ, Rodriguez-Mañas

L. Diabetes and frailty: two converging conditions? Can J Diabetes. 2016;40(1):77-83.

36. Feng L, Roiland R, Chen DD, Qiu C. Linking cognition and frailty in middle and old age: metabolic syndrome matters. Int $\mathrm{J}$ Geriatr Psychiatry. 2015;30:64-71.

37. Barzilay JI, Blaum C, Moore T, et al. Insulin resistance and inflammation as precursors of frailty. Arch Intern Med. 2007;167:635-41.

38. Varadhan R, Walston J, Cappola AR, et al. Higher levels and blunted diurnal variation of cortisol in frail older women. J Gerontol Med Sci. 2008;63(2):190-5.

39. Azzopardi RV, Beyer I, Vermeiren $\mathrm{S}$, et al. Increasing use of cognitive measures in the operational definition of frailty: A systematic review. Ageing Res Rev. 2018;43:10-6.

40. Muller M, Grobbee D, Thijssen J, van den Beld A, van der Schouw Y. Sex hormones and male health: effects on components of the frailty syndrome. Trends Endocrinol Metab. 2003;14(6):289-96.

41. Rockwood K, Stadnyk K, MacKnight C, et al. A brief clinical instrument to classify frailty in elderly people. Lancet. 1999;353 (9148):205-6.

42. Rockwood K, Song X, MacKnight $\mathrm{C}$, et al. A global clinical measure of fitness and frailty in elderly people. Can Med Assoc J. 2005;173(5):489-95.

43. van Kan G, Rolland Y, Bergman $\mathrm{H}$, et al. The I.A.N.A. Task force on frailty assessment of older people in clinical practice. J Nutr Health Aging. 2008;12 (1):29-37.

44. van Kan GA, Rolland YM, Morley JE, Vellas B. Frailty: toward a clinical definition. J Am Med Dir Assoc. 2008;9(2):71-2. 
45. Morley JE, Malmstrom TK, Miller DK. A simple frailty questionnaire (FRAIL) predicts outcomes in middle aged african americans. J Nutr Heal Aging. 2012;16(7):601-8.

46. Gobbens RJJ, Luijkx KG, Wijnen-Sponselee MT, Schols JMG. Towards an integral conceptual model of frailty. J Nutr Health Aging. 2010;14(3):175-81.

47. Gómez JF, Curcio C-L, Alvarado B, Zunzunegui MV, Guralnik J. Validity and reliability of the Short Physical Performance Battery (SPPB): a pilot study on mobility in the Colombian Andes. Colomb Med. 2013;44(3):165-71.

48. Wennie Huang WN, Perera S, Vanswearingen J, Studenski S. Performance measures predict onset of activity of daily living difficulty in community-dwelling older adults. J Am Geriatr Soc. 2010;58(5):844-52.

49. Guralnik JM, Ferrucci L, Simonsick EM, Salive ME, Wallace RB. Lowerextremity function in persons over the age of 70 years as a predictor of subsequent disability. $\mathrm{N}$ Engl J Med. 1995;332(9):556-62.

50. Gil Gregorio P. Evaluación clínica y herramientas diagnósticas. Rev Esp Geriatr Gerontol. 2017;52 (Supl 1):34-8.

51. Abdulrab K, Heun R. Subjective memory impairment: a review of its definitions indicates the need for a comprehensive set of standardised and validated criteria. Eur Psychiatry. 2008;23(5):321-30.

52. Hughes CP, Berg L, Danziger WL, Coben LA, Martin RL. A new clinical scale for the staging of dementia. $\mathrm{Br} \mathrm{J}$ Psychiatry. 1982;140:566-72.

53. Won CW, Lee Y, Kim S, Yoo J, Kim M, Ng T-P, et al. Modified criteria for diagnosing "cognitive frailty". Psychiatry Investig. 2018;15(9):839-42.

54. Dedeyne L, Deschodt M, Vershueren S, Tournoy J, Gielen E. Effects of multi-domain interventions in (pre) frail elderly on frailty, functional, and cognitive status: a systematic review. Clin Interv un Aging. 2017;12:873-96.

55. Gómez Pavón J, Alcaraz López C, Cristoffori G, Aguado R, Ares Conde B. Concepto de fragilidad: detección y tratamiento. Tipología del paciente anciano. Valoración geriátrica integral y criterios de calidad asistencial. Medicine. 2018;12(62):3627-90.

56. Talegawkar SA, Bandinelli S, Bandeen-roche $\mathrm{K}$, et al. A Higher Adherence to a mediterranean-style diet is inversely associated with the development of frailty in communitydwelling elderly men and women. J Nutr. 2012;142 (12):2161-6.

57. Ngandu T, Lehtisalo J, Solomon A, et al. A 2 year multidomain intervention of diet, exercise, cognitive training, and vascular risk monitoring versus control to prevent cognitive decline in atrisk elderly people (FINGER): a randomised controlled trial. Lancet. 2015;385(9984):2255-63. 